\title{
Inibidores de Bomba de Prótons: vantagens e desvantagens do uso prolongado
}

\author{
Proton Pump Inhibitors: advantages and disadvantages of long-term use
}

Inhibidores de la Bomba de Protones: ventajas y desventajas del uso a largo plazo

Ludimilla Santos Araújo ${ }^{1 *}$, Beatriz Moura Araujo², Carolina Siqueira Guimarães ${ }^{3}$, Danielle Naara Silva Fróes ${ }^{1}$, Elen Carolina Silva Maia ${ }^{3}$, Gabriela Hoffmann ${ }^{4}$, Jennifer Nicole Padua Kinsler ${ }^{5}$, Tamara Ribeiro de Oliveira ${ }^{6}$, Túlio Ribeiro de Oliveira ${ }^{7}$, Nestor Augusto Avelino Leite ${ }^{8}$.

\section{RESUMO}

Objetivo: Descrever as principais indicações, bem como as possíveis desvantagens do uso prolongado dos Inibidores de Bomba de Prótons (IBPs), a partir de uma revisão narrativa da literatura atual. Revisão Bibliográfica: Os IBPs são fármacos que levam ao bloqueio da secreção gástrica, com a finalidade de aumentar o pH do suco gástrico, através da inibição da enzima $\mathrm{H}_{+} / \mathrm{K}_{+}-\mathrm{ATP}$ ase. Dessa forma, os IBPs são considerados eficazes, desde que ingeridos da forma correta, em tratamentos de distúrbios gastrointestinais, como a úlcera péptica. $O$ uso prolongado desses inibidores sem reavaliação médica contribui para 0 surgimento de efeitos adversos, bem como aumentar o risco de dependência, podendo levar, também, à Síndrome de Zollinger-Ellison (ZES) ou também chamada de hipergastrinemia, além de interações farmacocinéticas e polifarmácia. Considerações finais: Não há dúvidas acerca da eficácia dos IBPs no tratamento e prevenção de distúrbios gástricos. Entretanto, sua elevada prevalência de uso reforça a importância do manejo adequado e reavaliação médica, incluindo a desprescrição quando não houver indicação para o uso, de forma a reduzir a ocorrência dos efeitos adversos relacionados ao uso desse fármaco a longo prazo.

Palavras-chave: Ácido gástrico, Inibidores de bomba de prótons, Efeitos adversos de longa duração, Eficácia.

\begin{abstract}
Objective: To describe the main indications, as well as the possible disadvantages of the prolonged use of Proton Pumb Inhibitors (PPIs), based on a review of the current scientific literature. Literature review: PPIs are drugs that blocks gastric secretion, with the aim of increasing the $\mathrm{pH}$ of the gastric juice, through the inhibition of the enzyme $\mathrm{H}_{+} / \mathrm{K}_{+}-\mathrm{ATPase}$. Thus, PPIs are considered effective, as long as they are ingested correctly, in the treatment of gastrointestinal disorders, such as peptic ulcers. The prolonged inhibitors usage without medical reassessment contributes to the emergence of adverse effects, as well as increasing the risk of dependence, which can also lead to Zollinger-Ellison (ZES), also called Hypergastrinemia, in addition to pharmacokinetic interactions and polypharmacy. Final considerations: There is no doubt about the effectiveness of PPIs in the treatment and prevention of gastric disorders. However, its high prevalence of use reinforces the importance of proper management and medical reassessment, including deprescription when there is no indication for use, in order to reduce the occurrence of adverse effects related to the long-term use of this drug.
\end{abstract}

Key words: Gastric acid, Proton pump inhibitors, Long term adverse effects, Efficacy.

\footnotetext{
${ }^{1}$ Centro Universitário Atenas (UniAtenas), Paracatu - MG. *E-mail: ludinhasantos@hotmail.com

2 Instituto Tocantinense Presidente Antônio Carlos Porto (ITPAC-PORTO), Porto Nacional - TO.

${ }^{3}$ Faculdade de Minas (FAMINAS-BH), Belo Horizonte - MG.

${ }^{4}$ Universidade Comunitária da Região de Chapecó (Unochapecó), Chapecó - SC.

${ }^{5}$ Universidade Vila Velha (UVV), Vitória - ES.

${ }^{6}$ Universidade Municipal de São Caetano do Sul (USCS), São Caetano do Sul - SP.

${ }^{7}$ Faculdade de Medicina de Barbacena (FAME/FUNJOBE), Barbacena - MG.

${ }^{8}$ Faculdade de Medicina de Campos (FMC), Campos dos Goytacazes - RJ.
} 


\section{RESUMEN}

Objetivo: Describir las principales indicaciones, así como las posibles desventajas del uso prolongado de Inhibidores de la Bomba de Protones (IBP), a partir de una revisión narrativa de la literatura actual. Revisión de la literatura: Los IBP son fármacos que bloquean la secreción gástrica, con el propósito de incrementar el pH del jugo gástrico, mediante la inhibición de la enzima $\mathrm{H}_{+} / \mathrm{K}_{+}-\mathrm{ATPasa}$. Por tanto, los IBP se consideran eficaces, siempre que se ingieran correctamente, en el tratamiento de trastornos gastrointestinales, como las úlceras pépticas. El uso prolongado de estos inhibidores sin reevaluación médica contribuye a la aparición de efectos adversos, además de aumentar el riesgo de dependencia, que también puede conducir al Síndrome de Zollinger-Ellison (ZES) o también llamado hipergastrinemia, además de interacciones farmacocinéticas y polifarmacia. Consideraciones finales: No hay duda de la eficacia de los IBP en el tratamiento y prevención de los trastornos gástricos. Sin embargo, su alta prevalencia de uso refuerza la importancia del manejo adecuado y la reevaluación médica, incluida la deprescripción cuando no hay indicación de uso, con el fin de reducir la aparición de efectos adversos relacionados con el uso a largo plazo de este fármaco.

Palabras clave: Ácido gástrico, Inhibidores de la bomba de protones, Efectos adversos a largo plazo, Eficacia.

\section{INTRODUÇÃO}

Os Inibidores da Bomba de Prótons (IBPs) são fármacos que apresentam como mecanismo de ação a inibição da enzima $\mathrm{H}+/ \mathrm{K}+-$ ATPase no estômago, levando ao bloqueio da secreção gástrica. Fisiologicamente, a partir dos estímulos distintos de três substâncias, há a ativação da enzima desta enzima, uma bomba de prótons, acarretando a secreção de Ácido Clorídrico $(\mathrm{HCl})$ no lúmen estomacal ao ocorrer o processo da troca de hidrogênio e potássio, consumindo Adenosina Trifosfato (ATP). Diante disso, para aumentar o pH do suco gástrico, os fármacos IBP atuam impedindo a ação da enzima fundindo seu receptor com resíduos de cisteína. Após a inibição da bomba de prótons, não é possível a sua regeneração, e a produção de ácido poderá ser efetiva somente após a síntese de uma nova enzima, o que garante o potencial de ação do fármaco entre 24 a 48 horas de ação (MORSCHEL CF, et al., 2018).

Por serem a classe de medicamentos antagonistas da produção de ácido gástrico mais potente, os IBPs estão entre as drogas mais prescritas atualmente. Seis IBPs são aprovados pela a Food \& Drug Administration (FDA), agência federal do departamento de saúde dos Estados Unidos: Omeprazol, Lansoprazol, Dexlansoprazol, Esomeprazol, Pantoprazol e Rabeprazol. Esses fármacos são atualmente utilizados para tratar doenças do refluxo gastroesofágico, dispepsia, infecção por Helicobacter pylori, esofagite de refluxo, úlcera péptica, entre outras condições (BOGHOSSIAN TA, et al., 2017; BRISEBOIS S, et al., 2018).

Apesar da eficácia comprovada dessa categoria farmacológica, muitos trabalhos científicos expõem potenciais riscos causados pelo seu uso prolongado. Dentre eles, destacam-se a perda da densidade óssea e consequente risco de fratura, deficiência da vitamina B12, hipomagnesemia, deficiência de ferro, doença renal, demência e infecção por Salmonella, Campylobacter e Clostridium difficile (BRISEBOIS S, et al., 2018).

Por ser uma classe medicamentosa de redução de secreção de ácidos, seu uso prolongado, sem a devida reavaliação, contribui para a polifarmácia e está associado a frequentes interações medicamentosas, sendo que aproximadamente 25 a $70 \%$ dos consumidores fazem uso inadequado desses medicamentos (BOGHOSSIAN TA, et al., 2017). Dentre as diversas outras alterações clínicas, os pacientes expostos ao uso prolongado de IBPs também podem apresentar efeito rebound ao suspender o tratamento, ou seja, há o ressurgimento de sintomas que estavam suprimidos durante o uso do medicamento, acarretando em hipersecreção e, posteriormente, dependência (CUNHA N e MACHADO AP, et al., 2018).

Na população idosa, os IBPs são comumente utilizados para a minimização dos desconfortos alimentares através da supressão gástrica e a elevação do pH estomacal, possuindo sua eficácia comprovada em tratamentos de distúrbios relacionados ao aumento da acidez gástrica. No entanto, nessa população, cerca de $40 \%$ dos indivíduos usam IBPs sem a real necessidade e, aproximadamente, $60 \%$ desenvolvem dependência (COSTA SL, et al., 2021). Devido a ascensão da prescrição e do uso impróprio dessa categoria de medicamentos, manifesta-se a necessidade da ampliação do estudo dos efeitos do uso contínuo dos medicamentos IBPs (MORSCHEL CF, et al., 2018). 
Nessa perspectiva, e considerando a relevância do assunto, o presente trabalho teve como objetivo revisar e discutir as principais indicações e possíveis desvantagens do uso prolongado dos IBPs, a partir da análise da produção científica atual sobre o tema. A partir disso, protocolos podem ser melhor estruturados e novos estudos sobre a temática fomentados.

\section{REVISÃO BIBLIOGRÁFICA}

A produção de $\mathrm{HCl}$ no estômago acontece por meio da bomba de prótons, através da substituição de íons potássio $(\mathrm{K}+)$ e liberação de íons hidrogênio $\left(\mathrm{H}_{+}\right)$. Os IBPs atuam se ligando de forma irreversível a essa bomba por meio da ligação covalente, o que inibe a produção do HCl (NOVOTNY M, et al., 2019).

Esses medicamentos são pró fármacos, dessa forma devem ser administrados pelo menos 30 minutos antes da alimentação, pois esta irá estimular a produção da gastrina que ativará a bomba $\mathrm{H}_{+} / \mathrm{K}+$ ATPase, 0 que tornará o ambiente ácido e possibilitará a ativação do fármaco. Além disso, a meia vida plasmática dura entre uma e duas horas, sendo rapidamente absorvidos após a administração, com metabolização através das enzimas hepáticas do citocromo P450 (SALGADO AL, et al., 2019).

Os IBPs, em suas diferentes fórmulas, são ácidos fracos que compartilham a mesma estrutura básica de suas moléculas, diferenciando-se entre si apenas em seus radicais (MORSCHEL CF, et al., 2018). O primeiro medicamento da classe, o omeprazol, foi introduzido em 1989 e em seguida foram lançados o lansoprazol, rabeprazol, pantoprazol, esomeprazol e dexlansoprazol. O omeprazol e o pantoprazol são os medicamentos mais usuais, mas não, necessariamente, os mais eficazes. $O$ esomeprazol e o dexlansoprazol aparecem à margem contrária, exibindo indicativos farmacocinéticos melhores (BARBOSA MA, et al., 2020).

Os IBPs podem ser adquiridos sem receita médica em vários países e estima-se que até $10 \%$ da população mundial faz uso de algum tipo de IBP. No Brasil, essa classe tem o estatuto de medicamentos de prescrição obrigatória, mas é prática habitual que sejam vendidos em farmácias sem a exigência de prescrição médica, uma vez que fármacos são os supressores mais potentes da secreção de ácido gástrico, independentemente de outros fatores estimuladores (ARRAIS PD, et al., 2016; CAMILO SP, et al., 2020).

Esses medicamentos exercem um papel importante no manejo de doenças pépticas relacionadas à acidez gástrica. Assim, a terapia com os IBPs é indicada em situações clínicas como a doença ulcerosa péptica, na qual eles são considerados a droga anti-secretora de primeira linha para o tratamento; na Doença do Refluxo Gastroesofágico (DRGE), incluindo o tratamento da esofagite erosiva ou esôfago de Barrett; na síndrome de Zollinger-Ellison, onde os IBPs são utilizados frequentemente em altas doses, necessárias para controlar a hipersecreção de ácido proveniente dos tumores; na prevenção primária de úlceras gastroduodenais associadas ao uso de Anti-inflamatórios Não Esteroidais (AINEs); e na erradicação do Helicobacter pylori (ZATERKA S, et al., 2019; WOLFE MM, et al., 2020).

Além dos IBPs, existem também outras classes de medicamentos que atuam como antagonistas da produção do ácido gástrico, por exemplo os bloqueadores de $\mathrm{H} 2$, antiácidos e prostaglandinas, sendo também responsáveis por reduzirem a acidez gástrica. Eles são utilizados no tratamento de determinadas doenças como a infecção por Helicobacter pylori, úlcera péptica, doença de refluxo gastroesofágico e muitas outras formas de gastrite. Todos possuem diferentes formas de atuação no organismo (VAKIL NB, 2020).

Existem alguns fatores que prejudicam a resposta adequada dos IBPs, apesar da sua eficácia geral comprovada, como a meia-vida plasmática curta e a administração pré-prandial obrigatória. Isso leva, frequentemente, ao uso da medicação duas vezes ao dia para evitar surtos, principalmente à noite, devido a recuperação da liberação de ácido clorídrico. Ainda assim, vários pacientes permanecem com sintomas noturnos mesmo com a dosagem duplicada (STRAND DS, et. al., 2017).

Além disso, a literatura mostra que cerca de $50 \%$ dos pacientes não tomam os IBPs entre 30 e 60 minutos antes da primeira refeição do dia, conforme recomendado, e isso prejudica o efeito pleno do medicamento. Constatou-se, ainda, que fatores intrínsecos como distúrbios de motilidade no trato gastrointestinal, hipersensibilidade visceral e refluxo duodeno-gastro-esofágico também contribuem para essa limitação. Além 
disso, aproximadamente metade dos pacientes que possuem DRGE não erosiva continuam sintomáticos mesmo com o uso de IBP (STRAND DS, et. al., 2017).

Diante disso, vários esforços têm sido feitos para melhorar essa classe de medicamentos, como a implementação de tecnologias para prolongar a atividade dos IBPs convencionais e desenvolvimento de novos IBPs de longa duração. Além disso, têm sido estudadas medidas para evitar a necessidade da administração antes de refeições, como a associação de um estimulador de secreção ácido gástrico, como o ácido succínico, junto ao IBP. Um ensaio pré-clínico mostrou que a terapia combinada de ácido succínico associado ao omeprazol demonstrou um controle noturno do $\mathrm{pH}$ estomacal superior à terapia isolada com omeprazol (STRAND DS, et al., 2017; CHOWERS Y, et al., 2012).

Existem algumas situações crônicas que justificam o uso contínuo de IBPs. Porém, para a maioria dos pacientes cujos sintomas gastrintestinais são persistentes, é possível o tratamento intermitente com essas drogas. Assim, pesquisas têm reforçado acerca de possíveis riscos relacionados ao uso contínuo dos IBPs, sem que haja reavaliação médica, e que contribuem para a polifarmácia, maior risco de interação medicamentosa e eventos adversos (BRISEBOIS S, et al., 2018).

O uso crônico dos IBPs pode levar ao efeito rebound, que pode acontecer com todas as drogas supressoras da acidez gástrica, como antiácidos, antagonistas do receptor H2 e os IBPs. Estudos clínicos evidenciaram que houve hipersecreção ácida após a interrupção do uso de IBPs, principalmente quando ocorreu de forma abrupta. Em decorrência disso, muitos pacientes desenvolvem dependência dessas drogas, o que explica o grande aumento de prescrições de IBPs nos últimos anos e o uso prolongado indevidamente. Estudos têm mostrado que a utilização de IBPs sem prescrição adequada pode chegar a $65 \%$ dos indivíduos que utilizam esses medicamentos (HELGADOTTIR HH e BJORNSSON ES, 2019; CUNHA N e MACHADO AP, 2018).

Outro efeito da terapia a longo prazo é a hipergastrinemia causada por um feedback negativo entre o $\mathrm{pH}$ intragástrico e a célula G antral (CAMILO SP, et al., 2020). A hipergastrinemia aumenta o risco de desenvolver tumores carcinóides, devido a ação trófica da gastrina em excesso, levando a hiperplasia de células enterocromafins-like e hipertrofia de células parietais. Estudos de base populacional mostraram uma forte relação entre o uso indiscriminado dos IBPs a longo prazo e aumento da incidência de tumores carcinóides gástricos (CHEUNG KS, et al., 2018; BRUSSELAERS N, et al., 2017). Entretanto, não é bem estabelecido se a gastrina isoladamente induz ao câncer gástrico ou se age como um cofator de alterações pré-malignas já existentes (HELGADOTTIR H e BJORNSSON ES, 2019).

Pesquisas têm mostrado, ainda, que podem ocorrer interações farmacocinéticas dos IBPs com outros tipos de fármacos. Entre estes, cita-se os antiagregantes plaquetários como o clopidogrel, que apesar de ainda não terem evidências bem estabelecidas, é recomendado cautela na utilização a longo prazo dos IBPs. Essa recomendação é baseada na metabolização dos IBPs pela via do citocromo P450 2C19 (CYP2C19) o que pode afetar a conversão hepática do clopidogrel em seu metabólito ativo devido ao bloqueio dessa via (HELGADOTTIR H e BJORNSSON ES, 2019). Reforça-se, entretanto, que o lansoprazol, dexlansoprazol e pantoprazol possuem outras vias de metabolização da via CYP2C19, sendo recomendado a utilização preferencial de um deles em doentes que possuam riscos cardiovasculares e façam uso do clopidogrel (CUNHA N e MACHADO AP, 2018).

Alguns estudos recentes demonstraram uma interferência dos IBPs em antiagregantes plaquetários não dependentes da ativação pelo CYP2C19, como o ticagrelor e aspirina. Acredita-se que o mecanismo nesse caso ocorra devido a uma disfunção endotelial causada pelos IBPs que leva a diminuição de óxido nítrico. Dessa forma, é recomendado evitar o uso concomitante de IBPs e drogas que também causem hipomagnesemia, como digoxina e diuréticos (CUNHA N e MACHADO AP, 2018; STRAND DS, et al., 2017).

Outros possíveis riscos relacionados ao uso contínuo dos IBPs relacionam-se a prejuízos ao metabolismo ósseo com aumento do risco de fraturas, deficiências nutricionais, infecções, doença renal e demência. Além disso, sabe-se que o uso crônico e em altas doses dos IBPs, pode afetar a absorção de micronutrientes como o cálcio, magnésio, vitamina B12, entre outros (GRAHAM DY e TANSEL A, 2018; BRISEBOIS S, et al., 2018; BOGHOSSIAN TA, et al., 2017). 
Quanto ao cálcio, acredita-se que a redução da secreção de ácido causada pelo medicamento prejudica a absorção deste mineral, que pode relacionar-se à diminuição da densidade óssea e aumento do risco de fraturas (STRAND DS, et al., 2017; HATEMI I e ESATOGLU SN, 2017; BRISEBOIS S, et al., 2018; FOSSMARK R, et al., 2019). Mesmo esse mecanismo não sendo completamente elucidado, o FDA determinou que todos os fabricantes dessa medicação revisassem e incluíssem em sua descrição sobre 0 possível risco de fraturas de quadril, pulso e coluna quando usados mais de uma vez ao dia ou por tempo superior a um ano (STRAND DS, et al., 2017).

Uma outra preocupação acerca da absorção de nutrientes é em relação a Vitamina B12. O ácido gástrico é necessário para separar essa vitamina das proteínas dietéticas e esta clivagem é necessária para que, posteriormente, essa vitamina possa se ligar ao fator intrínseco e estar disponível para absorção. Desta forma, foi relatado que o risco desta inibição aumenta a partir de dois anos ou mais de uso de IBPs, contudo, concluiuse que não há evidência suficiente para que seja incluída como rotina de suplementação ou triagem, devendo essa conduta ser discutida entre médicos e pacientes (BRISEBOIS S, et al., 2018).

Caso exista a diminuição da absorção da vitamina B12, seus efeitos podem ser graves se não for revertida podendo levar a um quadro de declínio cognitivo e desenvolvendo a demência, afetando além de mentalmente, o estado físico e social do indivíduo acometido. Também é possível desenvolver danos neurológicos, anemia e outras complicações, desta forma, sendo de grande importância para a saúde. (COSTA SL, et al., 2021; LAM JR, et al., 2013). Outros estudos afirmam que a demência pode ocorrer também devido os IBPs atravessarem a barreira hematoencefálica causando acúmulo do peptídeo beta-amiloide, substância envolvida na doença de Alzheimer. Esse acúmulo pode ocorrer, pois os IBPs impedem que a substância seja degradada efetivamente ou devido interferência nas bombas de prótons das células fagocitárias do Sistema Nervoso, a Microglia, também responsável por conter o acúmulo do peptídeo betaamilóide (COSTA SL, et al., 2021).

O magnésio é outro micronutriente que cuja redução na absorção é bem documentada em pacientes em uso de IBPs, porém, essa ocorrência é rara e está mais relacionada a paciente em uso concomitante de diuréticos. Em 2011, o FDA, alertou sobre o uso prolongado dos IBPs e a hipomagnesemia, recomendando a rotina de investigação dos níveis para aqueles pacientes que farão tratamento prolongado e que utilizam digoxina ou outros diuréticos (FOSSMARK R, et al., 2019; STRAND DS, et al., 2017). Além dos riscos do uso concomitante de diuréticos para idades avançadas, pode haver, ainda, risco aumentado de hipomagnesemia em indivíduos portadores de insuficiência renal crônica (HATEMI I e ESATOGLU SN; 2017).

Outro importante nutriente que sofre alteração em seu metabolismo é o ferro alimentar. O ácido gástrico participa da conversão da forma de ferro férrico em ferroso, necessária para que ocorra a absorção desse nutriente. Assim, o uso de IBPs pode estar associado ao desenvolvimento de anemia, astenia e outras complicações relacionadas à deficiência de ferro (BRISEBOIS S, et al., 2018).

Em 1992, o uso dos IBPs também foi relacionado com o risco para doença renal aguda devido a ocorrência de um caso de necrose tubular, associando ao resultado de dois grandes estudos observacionais que relacionaram o IBP a Doença Renal Aguda (DRA) e Doença Renal Crônica (DRC), bem como a progressão de DRC para terminal (BRISEBOIS S, et al., 2018). O mecanismo dessa associação ainda não foi totalmente elucidado, mas considera-se que ao inibir as bombas de prótons gástricas, ocorre também a inibição da bomba de prótons tubular renal. Sabe-se, ainda, que os IBPs são considerados uma das causas mais comuns de Nefrite Intersticial Aguda (NIA) induzida por medicamentos (FOSSMARK R, et al., 2019).

Por fim, estudos também sugeriram que é possível que, ao reduzir-se a acidez estomacal, também é reduzida a proteção contra bactérias, uma vez que um baixo $\mathrm{pH}$ é bactericida para diversas bactérias entéricas como a Salmonella paratyphi, Salmonella Enteritidis e, em nível hospitalar, a Clostridium difficile. Dessa forma, estas pesquisas concluíram que há uma associação contundente entre o uso de IBPs e a ocorrência de infecções entéricas (ZATERKA S, et al., 2019; BRISEBOIS S, et al., 2018).

Assim, não há dúvidas acerca da eficácia dos IBPs no tratamento e prevenção de certas doenças, mas também é bem instituído os prejuízos do uso indevido e por longos períodos. Assim sendo, é necessário que 
haja uma abordagem minuciosa seguindo as melhores evidências disponíveis para que os benefícios do uso de IBP realmente superem os riscos, devendo ser desprescrito quando isso não acontecer (DHARMARAJAN TS, 2021).

\section{CONSIDERAÇÕES FINAIS}

A partir da análise dos achados da literatura, foi possível verificar as principais indicações e as desvantagens do uso prolongado dos IBPs. Uma vez que são fármacos de elevada prevalência de uso, estão, frequentemente, associados à dependência medicamentosa, polifarmácia, bem como consequências adversas devido ao uso prolongado. Dessa forma, reforça-se a necessidade de intervenções que visem priorizar as principais indicações e doses adequadas desses medicamentos, com base no tratamento de distúrbios gástricos. Além disso, o estímulo ao aprimoramento dos profissionais prescritores, bem como a orientação ao público das consequências atribuídas ao uso prolongado de IBPs poderiam ser medidas executadas, com o fito de reduzir os efeitos adversos provocados e garantir a aplicação correta destes fármacos.

\section{REFERÊNCIAS}

1. ARRAIS PD, et al. Prevalência da automedicação no Brasil e fatores associados. Revista de Saúde Pública, 2016;50 (supl 2):1-11.

2. BARBOSA MA, et al. Análise do impacto da similaridade farmacocinética dos inibidores da bomba de prótons. Revista de Casos e Consultoria, 2020; 11(1): e11133.

3. BOGHOSSIAN TA, et al. Deprescribing versus continuation of chronic proton pump inhibitor use in adults. Cochrane Database of Systematic Reviews, 2017; 3(3): CD011969.

4. BRISEBOIS S, et al. Proton pump inhibitors: Review of reported risks and controversies. Laryngoscope Investigative Otolaryngology, 2018; 3(6): 457-462.

5. BRUSSELAERS N, et. al. Maintenance therapy with proton pump inhibitors and risk of gastric cancer: A nationwide population-based cohort study in Sweden. BMJ Open, 2017; 7(10): e017739.

6. CAMILO SP, et. al. Uso crônico de inibidores de bomba de prótons e a quantidade de células $\mathrm{g}$, d e ecl no estômago. Arquivos Brasileiros de Cirurgia Digestiva, 2020;33(2):e1506.

7. CHEUNG KS, et. al. Long-term proton pump inhibitors and risk of gastric cancer development after treatment for Helicobacter pylori: A population-based study. Gut, 2018; 67: 28-35.

8. CHOWERS Y, et. al. The effect of once daily omeprazole and succinic acid (VECAM) vs once daily omeprazole on 24-h intragastric pH. Neurogastroenterology and Motility, 2012;24:426- 431.

9. COSTA SL, et al. Efeitos do uso prolongado de Inibidores de Bomba de Prótons em idosos. Brazilian Journal of Health Review, 2021; 4(2): 4248-4265.

10. CUNHA N, MACHADO AP. Inibidores da bomba de protões e o risco de eventos adversos graves-uma bomba cardiovascular?. Revista Portuguesa de Cardiologia, 2018; 37(10): 859-863.

11. DHARMARAJAN TS.The Use and Misuse of Proton Pump Inhibitors: An Opportunity for Deprescribing. Journal of the American Medical Directors Association, 2021; 22(1):15-22.

12. FOSSMARK R, et al. Adverse effects of proton pump inhibitors-evidence and plausibility. International journal of molecular sciences, 2019; 20: 5203.

13. GRAHAM DY, TANSEL A. Interchangeable use of proton pump inhibitors based on relative potency. Clinical Gastroenterology and Hepatology, 2018; 16(6); 800-808.

14. HATEMI İ, ESATOGLU SN. What is the long term acid inhibitor treatment in gastroesophageal reflux disease? What are the potential problems related to long term acid inhibitor treatment in gastroesophageal reflux disease? How should these cases be followed. The Turkish Journal of Gastroenterology, 2017; 28(1): 57-60.

15. HELGADOTTIR HH, BJORNSSON ES. Problems Associated with Deprescribing of Proton Pump Inhibitors. International Journal of Molecular Sciences. 2019, 20(21): 5469.

16. LAM JR, et al. Proton pump inhibitor and histamine 2 receptor antagonist use and vitamin B12 deficiency. JAMA, 2013; 310(22): 2435-2442.

17. MORSCHEL CF, et al. Inibidores da bomba de prótons e sua relação com a doença renal. Brazilian Journal of Nephrology, 2018;40(3): 301-306.

18. NOVOTNY M, et al. PPI long term use: Risk of neurological adverse events? Frontiers in Neurology, $2019 ; 9: 1142$.

19. SALGADO AL, et al. Uso indiscriminado de inibidores da bomba de prótons em receituários de medicamentos de uso contínuo. Brazilian Journal of Health Review, 2019; 2: 5883-5897.

20. STRAND DS, et al. 25 years of proton pump inhibitors: a comprehensive review. Gut and liver, $2017 ; 11(1): 27-37$.

21. VAKIL N, Antiulcer medications: Mechanism of action, pharmacology, and side effects. 2020. Disponível em: https://www.uptodate.com/contents/antiulcer-medications-mechanism-of-action-pharmacology-and-side-effects. Acesso em 2 de agosto de 2021.

22. WOLFE MM, et al. Proton pump inhibitors: Overview of use and adverse effects in the treatment of acid related disorders. 2020. Disponível em: https://www.uptodate.com/contents/proton-pump-inhibitors-overview-of-use-andadverse-effects-in-the-treatment-of-acid-related-disorders. Acesso em: 2 de agosto de 2021.

23. ZATERKA $S$, et al. Historical perspective of gastroesophageal reflux disease clinical treatment. Arquivos de gastroenterologia, 2019; 56(2): 202-208. 